\title{
The electromagnetic form factor of the pion in two-flavour lattice QCD
}

\author{
Bastian B. Brandt* \\ Institut für theoretische Physik, \\ Universität Regensburg, D-93040 \\ E-mail: bastian.brandt@physik. uni-regensburg.de
}

\section{Andreas Jüttner}

School of Physics and Astronomy,

University of Southampton, Southampton, SO14 1BJ, UK

\section{Hartmut Wittig}

PRISMA Cluster of Excellence, Institut für Kernphysik and Helmholtz Institut Mainz,

Johannes Gutenberg-Universität Mainz, D-55099

\begin{abstract}
We present the current status of our lattice calculation of the electromagnetic form factor of the pion with two flavours of non-perturbatively $\mathrm{O}(\mathrm{a})$-improved Wilson fermions. Using twisted boundary conditions and stochastic sources we obtain accurate results with a fine momentum resolution near $q^{2}=0$. This enables the computation of the charge radius without model dependence. The ensembles cover various lattice spacings and pion masses, ranging down to $250 \mathrm{MeV}$. This allows to compare the data to continuum chiral perturbation theory to NNLO including corrections of finite lattice spacing to perform a simultaneous chiral and continuum extrapolation. An estimate for the systematic error resulting from the extrapolation can be obtained by looking at the spread of results obtained from other functional forms such as polynomials.
\end{abstract}

Xth Quark Confinement and the Hadron Spectrum

8-12 October 2012

TUM Campus Garching, Munich, Germany

\footnotetext{
*Speaker.
} 


\section{Introduction}

Lattice QCD has by now entered the era in which high precision calculations of physical quantities at the physical point including dynamical fermionic degrees of freedom become feasible (see for example the quantities summarised in the FLAG review [1]). This progress has been possible largely due to substantial algorithmic improvements and the associated acceleration of the simulations allowing to simulate light quarks. Despite these successes there are quantities where a satisfactory control over systematics has not been achieved so far, among them quantities related to the structure of hadrons [2,3]. For the pion electromagnetic form factor the dominant systematic uncertainty is due to the chiral extrapolation. The form factor has been worked out in chiral perturbation theory $[4,5](\chi \mathrm{PT})$ to next-to-next-to leading order $[6,7](\mathrm{NNLO})$ which can be used to guide the chiral extrapolation. However, the pion mass range for which $\chi \mathrm{PT}$ to a given order is applicable is not known a priori.

In this proceedings article we present the status of our calculation of the pion form factor $f_{\pi \pi}\left(Q^{2}\right)$ in the region of small space-like momentum transfers $Q^{2}=-q^{2}$ in Lattice QCD with two dynamical quark flavours. In this regime the form factor encodes information about the electromagnetic charge radius $\left\langle r_{\pi}^{2}\right\rangle$ of the pion,

$$
f_{\pi \pi}\left(q^{2}\right)=1-\frac{1}{6}\left\langle r_{\pi}^{2}\right\rangle q^{2}+\mathscr{O}\left(q^{4}\right), \quad\left\langle r_{\pi}^{2}\right\rangle=\left.6 \frac{f_{\pi \pi}\left(q^{2}\right)}{q^{2}}\right|_{q^{2}=0} .
$$

Determinations of the charge radius usually suffer from an intrinsic model dependence since the slope of the form factor is obtained from fits over a large $Q^{2}$-interval assuming a particular model for the $Q^{2}$-dependence such as pole dominance. Here we use suitably tuned partially twisted boundary conditions $[8,9,10,11,12]$ to compute data points in the immediate vicinity of $Q^{2}=0$ which enables the model-independent extraction of the charge radius. The data is then compared to $\chi \mathrm{PT}$ which is used to extrapolate the charge radius to the physical point. The continuum extrapolation is done by introducing terms that model the cutoff dependence into the chiral extrapolation. The systematic uncertainty which is introduced by relying on $\chi \mathrm{PT}$ for our range of pion masses is taken into account by checking the results from $\chi \mathrm{PT}$ with results from a polynomial extrapolation. Parts of the results were already reported in $[13,14,15,16]$.

\section{Extraction of the form factor}

The electromagnetic form factor in two-flavour QCD is defined by

$$
\left\langle\pi^{+}\left(\vec{p}_{f}\right)\left|\frac{2}{3} \bar{u} \gamma_{\mu} u-\frac{1}{3} \bar{d} \gamma_{\mu} d\right| \pi^{+}\left(\vec{p}_{i}\right)\right\rangle=\left(p_{f}+p_{i}\right)_{\mu} f_{\pi \pi}\left(q^{2}\right) .
$$

In lattice QCD the matrix element can be extracted from suitable ratios of 2- and 3-point functions, in particular we use the ratio [12]

$$
R_{1}\left(t, \vec{p}_{i}, \vec{p}_{f}\right)=Z_{V}^{\mathrm{eff}} 4 \sqrt{E\left(\vec{p}_{i}\right) E\left(\vec{p}_{f}\right)} \sqrt{\frac{C_{3}\left(t, \vec{p}_{i}, \vec{p}_{f}\right) C_{3}\left(t, \vec{p}_{f}, \vec{p}_{i}\right)}{C_{2}\left(T / 2, \vec{p}_{i}\right) C_{2}\left(T / 2, \vec{p}_{f}\right)}},
$$




\begin{tabular}{ccccccc}
\hline \hline$\beta$ & $r_{0} / a$ & lattice & $m_{\pi}[\mathrm{MeV}]$ & $m_{\pi} L$ & Labels & Statistic \\
\hline 5.20 & $6.15(6)$ & $64 \times 32^{3}$ & $455,351,299$ & $6.0-4.0$ & A3 - A5 & $\mathscr{O}(100)$ \\
\hline 5.30 & $7.26(7)$ & $64 \times 32^{3}$ & 552,412 & $6.2,4.7$ & E4, E5 & $\mathscr{O}(100)$ \\
& & $96 \times 48^{3}$ & 295,254 & $5.0,4.2$ & F6, F7 & $\mathscr{O}(250)$ \\
\hline 5.50 & $10.00(11)$ & $96 \times 48^{3}$ & $625,534,424$ & $7.7-5.3$ & N3- N5 & $\mathscr{O}(100)$ \\
\hline \hline
\end{tabular}

Table 1: Compilation of simulation parameters.

where

$$
Z_{V}^{\mathrm{eff}}=\frac{C_{2}(T / 2, \overrightarrow{0})}{2 C_{3}(t, \overrightarrow{0}, \overrightarrow{0})}
$$

ensures the physical normalisation $f_{\pi \pi}(0)=1$ and

$$
\begin{aligned}
& C_{2}(t, \vec{p})=\sum_{\vec{x}_{i}, \vec{x}} e^{i \vec{p} \cdot\left(\vec{x}-\vec{x}_{i}\right)}\left\langle P(t, \vec{x}) P\left(0, \vec{x}_{i}\right)\right\rangle, \\
& C_{3}\left(t, \vec{p}_{i}, \vec{p}_{f}\right)=\sum_{\vec{x}_{i}, \vec{x}_{f}, \vec{x}} e^{i \vec{p}_{f} \cdot\left(\vec{x}_{f}-\vec{x}\right)} e^{i \vec{p}_{i} \cdot\left(\vec{x}-\vec{x}_{i}\right)}\left\langle P\left(t_{f}, \vec{x}_{f}\right) V_{0}^{I}(t, \vec{x}) P\left(0, \vec{x}_{i}\right)\right\rangle .
\end{aligned}
$$

Here $P(x)$ and $V_{\mu}^{I}(x)$ are the pseudoscalar density and the $\mathscr{O}(a)$-improved vector current, respectively, and $E(\vec{p})$ is the energy of the pion with momentum $\vec{p}$. Up to corrections from excited states the ratio in eq. (2.2) is constant in Euclidean time and gives the desired matrix element of the vector current from eq. (2.1). In practice, the factor $Z_{V}^{\text {eff }}$ is obtained prior to the extraction of the matrix element via eq. (2.3).

\section{Simulation setup}

The calculations are based on the CLS ensembles that have been generated with two degenerate flavours of non-perturbatively $\mathscr{O}(a)$-improved Wilson fermions using the deflation accelerated DDHMC [17] and MP-HMC [18] algorithms. The bare parameters and some of the basic properties of the lattices are given in table 1. We express dimensionful quantities in units of the Sommer scale $r_{0}$ [19], which was recently determined on the CLS ensembles $[20,21]$ with a physical value of $r_{0}=0.503(10) \mathrm{fm}[21]$.

All 2- and 3-point functions were computed using two hits of stochastic $Z_{2} \times Z_{2}$ wall sources [22, $23,24,25]$ on two different timeslices separated in time by $T / 2$. The only exception is ensemble A5 where we have used four source positions separated by $T / 4$. We are primarily interested in the region of small momentum transfers which is made accessible by partially twisted boundary conditions at vanishing Fourier momentum. For each ensemble we have used five different twist angles in the $x$-direction, that are tuned to achieve a high resolution in the low $Q^{2}$ region. In addition to the form factor we have also computed the renormalised PCAC quark mass $\hat{m}$ and pion decay constant $F_{\pi}$. All quantities are fully $\mathscr{O}(a)$-improved, and we adopt the renormalisation constants from $[26,27,28,29,21]$. The results are listed in table 2 . Note that our ensembles cover a large range of quark masses and lattice spacings, always satisfying $m_{\pi} L \geq 4$.

Statistical errors have been estimated using the bootstrap procedure with 1000 bins. At present all fits for the extraction of the masses and matrix elements are uncorrelated. 


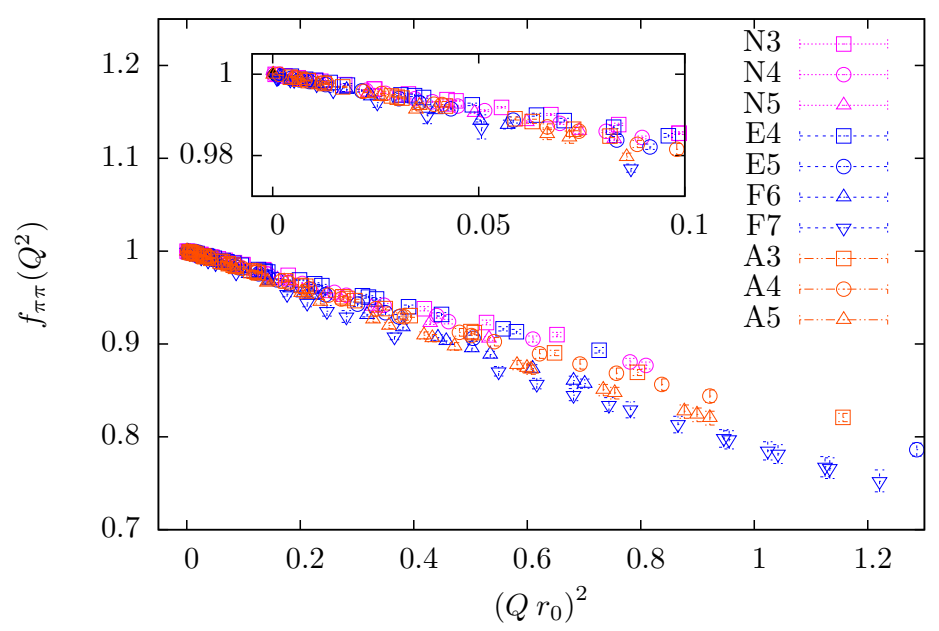

Figure 1: Results for the form factor on all ensembles. The inset shows a zoom in the region of low momentum transfer.

\section{Results for form factor and charge radius}

The full set of results for the form factor is shown in figure 1. The plot demonstrates that partially twisted boundary conditions are a powerful tool to explore the small momentum transfer region of form factors. In particular, the high density of points in the low $Q^{2}$ region enables the accurate and model independent extraction of the charge radius, as discussed below.

The data is still subject to effects stemming from the finite box size, even though they are expected to be small, since $m_{\pi} L \geq 4$. We estimate their size in $\chi \mathrm{PT}$. For the pion mass and the decay constant the relevant expressions have been derived in [30]. For the form factor with partially twisted boundary conditions, finite size effects are only known for the case where one of the twists is zero [31], i.e. either the initial twist $\theta_{i}=0$ or the final twist $\theta_{f}=0$, and in the Breit frame [32] where $\theta_{i}=-\theta_{f}$. As input for the $\chi \mathrm{PT}$ formulae NLO low energy constants (LECs) are needed. To become independent of external input we apply an iterative procedure: First we estimate the finite volume effects using the LECs from [33] as input. We then perform the extraction of the charge radius and the form factor and fit the data to NNLO $\chi$ PT. This yields new LECs that are used for the next iteration. After two such iteration this procedure converged and we did not see any residual deviations in the LECs.

From now on we constrain the data to those kinematical situations where finite size effects are known in $\chi$ PT. To extract the charge radius we compare the results from linear fits to the results for the slope at $Q^{2}=0$ obtained from polynomial fits to order two and three up to some maximal value of $Q^{2}$. The results from those different fits for ensemble F6 are shown in figure 2 (left). We also show the results obtained from the vector pole dominance model, i.e. of fits to a single pole form, which has predominantly been used to extract the charge radius from the data of the form factor. Surprisingly, the linear fit starts to deviate from the other functional forms at relatively small values of $Q^{2}$, which shows that higher order terms become important already around $\left(Q r_{0}\right)^{2} \approx 0.1$. Since we like to extract the charge radius using a fit to the same $Q^{2}$ interval for all ensembles 


\begin{tabular}{lcccc}
\hline \hline ensemble & $r_{0} m_{\pi}$ & $r_{0} F_{\pi}$ & $r_{0} \hat{m}$ & $Z_{V}^{\text {eff }}$ \\
\hline \hline A3 & $1.161(12)$ & $0.280(8)$ & $0.090(3)$ & $0.73228(7)$ \\
A4 & $0.895(11)$ & $0.247(12)$ & $0.052(3)$ & $0.72885(12)$ \\
A5 & $0.761(11)$ & $0.251(14)$ & $0.040(2)$ & $0.72731(10)$ \\
\hline E4 & $1.406(16)$ & $0.287(10)$ & $0.128(5)$ & $0.74962(8)$ \\
E5 & $1.048(13)$ & $0.271(11)$ & $0.078(4)$ & $0.74461(8)$ \\
F6 & $0.752(8)$ & $0.254(8)$ & $0.041(2)$ & $0.74119(4)$ \\
F7 & $0.646(7)$ & $0.237(8)$ & $0.029(1)$ & $0.74030(5)$ \\
\hline N3 & $1.593(18)$ & $0.329(7)$ & $0.188(5)$ & $0.77162(3)$ \\
N4 & $1.360(16)$ & $0.304(9)$ & $0.139(4)$ & $0.76855(3)$ \\
N5 & $1.080(13)$ & $0.291(8)$ & $0.091(3)$ & $0.76543(3)$ \\
\hline \hline
\end{tabular}

Table 2: Results for basic quantities.
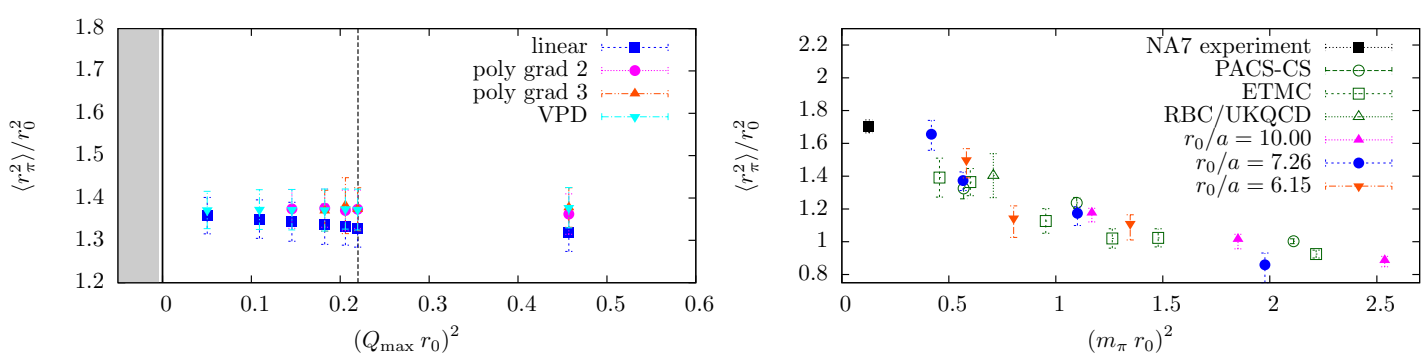

Figure 2: Left: The squared charge radius versus the maximal value of $Q^{2}$ entering the fit at a pion mass of $295 \mathrm{MeV}$ (ensemble F6). Shown are the results from a linear fit, polynomial fits of degrees two and three, and the results of a fit to the vector pole dominance model (VPD). Right: Compilation of results for the charge radius in dynamical lattice QCD [35, 36, 37] and from experiment [34].

we have to use the fit to a polynomial of degree two with a maximal value of $\left(Q r_{0}\right)^{2}=0.22$, as indicated by the vertical dashed line in figure 2 (left). In this case all ensembles have more than three data points for the form factor below this $Q^{2}$-cut. Note, that this does not affect the model independent extraction of the charge radius, since we can explicitly check for the other ensembles that the results are consistent with the ones from a linear fit to the very small $Q^{2}$-region. The results for the charge radius are shown in comparison to results from experiment [34] and other collaborations $[35,36,37]$ in figure 2 (right).

\section{Chiral and continuum extrapolation}

The remaining task is the extrapolation to the physical point. The natural framework for the chiral extrapolation is $S U(2) \chi \mathrm{PT}$. Since we have found that $\chi \mathrm{PT}$ to next-to-leading order (NLO) does not work for our range of pion masses [15] (for more details see also our upcoming publication), it is necessary to use $\chi \mathrm{PT}$ to NNLO. The expression for the form factor at NNLO in continuum $\chi$ PT has been worked out in [6].

For the continuum extrapolation we have to keep track of lattice artefacts, entering at order $a^{2}$. To take these into account we include a term proportional to $Q^{2} a^{2}$, which respects the normalisation 
$f_{\pi \pi}(0)=1$. The resulting formulae then contain 6 free parameters and not all of them can be constrained sufficiently by a fit to the data for the form factor alone. To overcome this problem we perform a simultaneous chiral extrapolation of $f_{\pi \pi}\left(Q^{2}\right)$ (or directly $\left\langle r_{\pi}^{2}\right\rangle$ ) together with $F_{\pi}$ and $m_{\pi}$ with respect to the isospin-averaged light quark mass $\hat{m}$. An account of the fit formulae will be given in our future publication. ${ }^{1}$ The inclusion of the additional quantities further constrains some of the LECs that are less constrained by the $m_{\pi}$ and $Q^{2}$-dependence of the form factor. In total the formulae including terms modeling cutoff effects now contain 14 free parameters and further input is needed to be able to obtain reliable results. We therefore decided to fix the LECs $\bar{\ell}_{1}=-0.4(5)$ and $\bar{\ell}_{2}=4.3(1)$ by $\pi \pi$-scattering [33]. We have verified explicitly that our fit results are not very sensitive to $\bar{\ell}_{1}$ and $\bar{\ell}_{2}$ by checking that the results for $\left\langle r_{\pi}^{2}\right\rangle$ at the physical point do not change significantly even if we vary them by a factor two.

To eventually perform the chiral extrapolation we have used three different procedures, i.e.

- simultaneous fits to $\chi \mathrm{PT}$ to NNLO including $f_{\pi \pi}\left(Q^{2}\right), F_{\pi}$ and $m_{\pi}$,

- simultaneous fits to $\chi \mathrm{PT}$ to NNLO including $\left\langle r_{\pi}^{2}\right\rangle, F_{\pi}$ and $m_{\pi}$,

- polynomial fits to $\left\langle r_{\pi}^{2}\right\rangle$.

The last two procedures make use of the model independent extraction of the charge radius discussed in the previous section while the first procedure assumes that $\chi \mathrm{PT}$ also provides the correct $Q^{2}$-dependence of the form factor. Thus the last two procedures are the preferred choices for obtaining a model independent result for the charge radius at the physical point. The polynomial fits provide estimates for the systematic effects stemming from the assumption that $\chi \mathrm{PT}$ to NNLO provides the correct functional form for the chiral extrapolation for the present range of pion masses. An estimate for the associated error can be obtained from the spread of the results of the three different procedures.

Figure 3 shows an example for the chiral extrapolation using $\chi \mathrm{PT}$ to NNLO for $\left\langle r_{\pi}^{2}\right\rangle, F_{\pi}$ and $m_{\pi}$ including only ensembles with a pion mass below $600 \mathrm{MeV}$. The fit describes the data well and yields an uncorrelated $\chi^{2} /$ dof $=1.3$ with 11 free parameters. The result in the continuum (red band) and at the physical point for the charge radius is larger than the experimental value, but not significantly. The result for the the pion decay constant is consistent with the experimental result. The same is true for the LECs that are in good agreement with the typical results found in the literature. A more comprehensive discussion of the chiral extrapolations will be given in our future publication.

\section{Conclusions}

In this proceedings article we have given an account of our comprehensive study of the electromagnetic form factor of the pion at small momentum transfers in lattice QCD. The high density of accurate data points close to $Q^{2}=0$ allows for the model independent extraction of the charge radius at finite lattice spacings. Finite volume effects have explicitly been studied and corrected using $\chi \mathrm{PT}$ to NLO and all quantities are fully $\mathscr{O}(a)$-improved. While $\chi \mathrm{PT}$ to NLO fails to describe

\footnotetext{
${ }^{1}$ The $\chi$ PT part of the formulae is identical with the ones given in [36].
} 

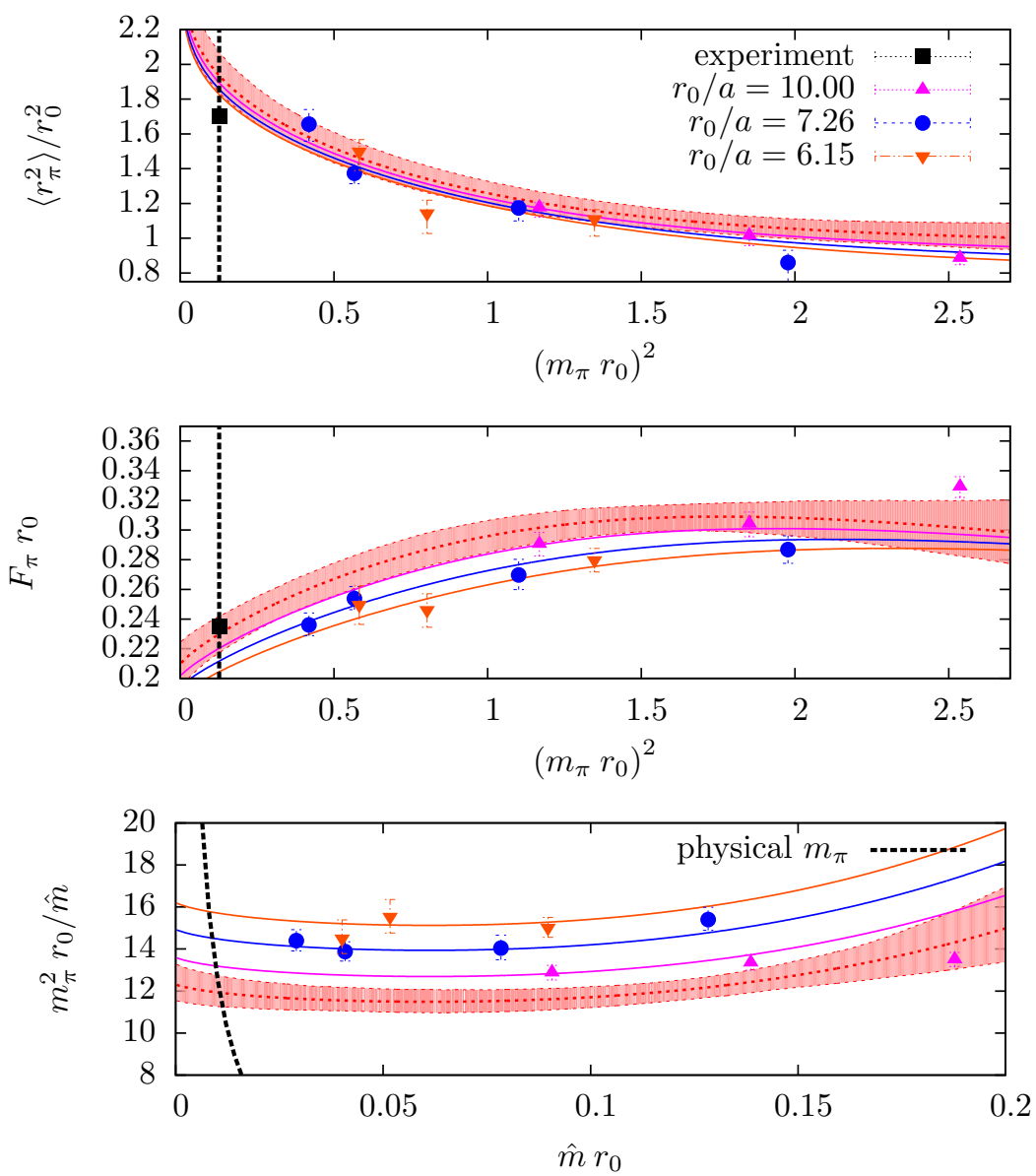

Figure 3: Results of a simultaneous fit to the data for $\left\langle r_{\pi}^{2}\right\rangle$, as extracted in the previous section, $F_{\pi}$ and $m_{\pi}$ using $\chi \mathrm{PT}$ to NNLO with pion masses below $\left(m_{\pi} r_{0}\right)^{2}=2$. The coloured dashed lines are the central values for the different lattice spacings and the red band and the red dashed line represent the error band and the central value in the continuum.

the data consistently in this range of pion masses, $\chi$ PT to NNLO provides a good description. However, stabilising the fits is challenging, which agrees with what has been found in $[35,36]$. Final numbers and detailed discussions will be given in our upcoming publication.

\section{Acknowledgments}

We are grateful to our colleagues within CLS for sharing gauge ensembles. The calculations of the correlation functions were performed on the dedicated QCD platform "Wilson" at the Institute for Nuclear Physics, University of Mainz. We thank Dalibor Djukanovic for technical support. The work was supported by DFG (SFB443) and the Research Center EMG funded by Forschungsinitiative Rheinland-Pfalz.

\section{References}

[1] G. Colangelo et al., Eur. Phys. J. C 71 (2011) 1695 [arXiv:1011.4408] 
[2] D. B. Renner, PoS QNP 2012 (2012) 003 [arXiv: 1207.2680]

[3] H. -W. Lin, PoS LATTICE 2012 (2012) 013 [arXiv:1212.6849]

[4] J. Gasser and H. Leutwyler, Nucl. Phys. B 250 (1985) 465

[5] J. Gasser and H. Leutwyler, Nucl. Phys. B 250 (1985) 517

[6] J. Bijnens, G. Colangelo and P. Talavera, JHEP 9805 (1998) 014 [hep-ph/9805389]

[7] J. Bijnens and P. Talavera, JHEP 0203 (2002) 046 [hep-ph/0203049]

[8] P. F. Bedaque, Phys. Lett. B 593 (2004) 82 [nucl-th/0402051]

[9] C. T. Sachrajda and G. Villadoro, Phys. Lett. B 609 (2005) 73 [hep-lat/0411033]

[10] G. M. de Divitiis, R. Petronzio and N. Tantalo, Phys. Lett. B 595 (2004) 408 [hep-lat/0405002]

[11] J. M. Flynn et al. [UKQCD Collaboration], Phys. Lett. B 632 (2006) 313 [hep-lat/0506016]

[12] P. A. Boyle et al, JHEP 0705 (2007) 016 [hep-lat/0703005]

[13] B. B. Brandt et al., PoS LATTICE 2010 (2010) 164 [arXiv:1010.2390]

[14] B. B. Brandt et al., Eur. Phys. J. ST 198 (2011) 79 [arXiv:1106.1554]

[15] B. B. Brandt, A. Jüttner and H. Wittig, arXiv:1109.0196

[16] B. B. Brandt et al, Prog. Part. Nucl. Phys. 67 (2012) 223

[17] M. Luscher, JHEP 0712 (2007) 011 [arXiv:0710.5417]

[18] M. Marinkovic and S. Schaefer, PoS LATTICE 2010 (2010) 031 [arXiv:1011.0911]

[19] R. Sommer, Nucl. Phys. B 411 (1994) 839 [hep-lat/9310022]

[20] B. Leder et al. [ALPHA Collaboration], PoS LATTICE 2010 (2010) 233 [arXiv:1012.1141]

[21] P. Fritzsch et al., Nucl. Phys. B 865 (2012) 397 [arXiv:1205.5380]

[22] M. Foster et al. [UKQCD Collaboration], Phys. Rev. D 59 (1999) 074503 [hep-lat/9810021]

[23] C. McNeile et al. [UKQCD Collaboration], Phys. Rev. D 73 (2006) 074506 [hep-lat/0603007]

[24] P. A. Boyle et al, JHEP 0808 (2008) 086 [arXiv:0804.1501]

[25] E. Endress, A. Juttner and H. Wittig, arXiv: 1111.5988

[26] M. Della Morte et al. [ALPHA Collaboration], Nucl. Phys. B 729 (2005) 117 [hep-lat/0507035]

[27] M. Della Morte, R. Hoffmann and R. Sommer, JHEP 0503 (2005) 029 [hep-lat/0503003]

[28] M. Della Morte, R. Sommer and S. Takeda, Phys. Lett. B 672 (2009) 407 [arXiv:0807.1120]

[29] P. Fritzsch, J. Heitger and N. Tantalo, JHEP 1008 (2010) 074 [arXiv:1004.3978]

[30] G. Colangelo, S. Dürr and C. Haefeli, Nucl. Phys. B 721 (2005) 136 [hep-lat/0503014]

[31] F. -J. Jiang and B. C. Tiburzi, Phys. Lett. B 645 (2007) 314 [hep-lat/0610103]

[32] F. -J. Jiang and B. C. Tiburzi, Phys. Rev. D 78 (2008) 037501 [arXiv:0806.4371]

[33] G. Colangelo, J. Gasser and H. Leutwyler, Nucl. Phys. B 603 (2001) 125 [hep-ph/0103088]

[34] S. R. Amendolia et al. [NA7 Collaboration], Nucl. Phys. B 277 (1986) 168

[35] O. H. Nguyen et al, JHEP 1104 (2011) 122 [arXiv:1102.3652]

[36] R. Frezzotti et al. [ETM Collaboration], Phys. Rev. D 79 (2009) 074506 [arXiv:0812.4042]

[37] P. A. Boyle et al, JHEP 0807 (2008) 112 [arXiv:0804.3971] 